\title{
On the mechanism of bluff body flow control by pulsed plasma actuator
}

\author{
Takashi MATSUNO*, Kengo MAEDA*, Noboru FUJITA*, Kenichi HARUNA*, \\ Gouji YAMADA* and Hiromitsu KAWAZOE* \\ * Department of Mechanical and Aerospace Engineering, Tottori University \\ 4-101 Koyama-Minami, Tottori-shi, Tottori 680-8552, Japan \\ E-mail: matsuno@mech.tottori-u.ac.jp
}

Received 30 January 2014

\begin{abstract}
The flow control performance of the pulse-actuated plasma actuator and their mechanism was investigated. To clarify the flow mechanism of the separation control on a circular cylinder when pulsed plasma actuator is applied, the characteristics of the induced jet and their influence on the separated flow were separately examined. The characteristics of the induced jet from the pulsed plasma actuator were examined from the results of jet thrust measurement and high-speed Schlieren flow visualization. The effect of the frequency of pulsed plasma actuator on the flow separation on a circular cylinder model is examined in the viewpoint if aerodynamic force and the flow field measured with time-resolved PIV at the low speed wind tunnel test. For the induced jet analysis, the strength of the induced jet is found to be widely varied with the modulation frequency. When the modulation frequency is high, over $250 \mathrm{~Hz}$, the thrust of the induced jet increases with the modulation frequency increased. For the separation control test, the drag is significantly dropped with increasing the modulation frequency. From these results, it turns out that the enhancement of the induced jet generated from plasma actuator in high modulation frequency regime is the main cause of the enhancement of drag reduction performance.
\end{abstract}

Key words : Low speed flow, Separation, Flow control, Plasma actuator, Jet, Circular cylinder

\section{Introduction}

In recent days, an application of plasma actuator receives increasing attention. Plasma actuator is an active flow control device which utilizes the weakly ionized air generated from the dielectric barrier discharge (DBD). The advantages of the plasma actuator are that fully electronic driven with no moving parts, simplicity in structure, fast response, and ability to simulate their effect in numerical flow solvers (Corke, et al., 2007).

Because of these advantages plasma actuator has been applied to various flow problems and shows their feasibility in the area such as separation control (Matsuno, 2010; Matsuno, et al., 2012, 2013; Segawa, et al., 2011). In our research group, several studies have been carried out on the flow control mechanism of plasma actuator and development of the high performance plasma actuator (Matsuno, et al., 2011).

Our current research is focusing on a suppression separation of the boundary layer on the circular cylinder model using the SDBD plasma actuators. For the cylinder wake control, numerous researches have been conducted over the years. They show the feasibilities of the active flow control device on this problem, as well as the dependencies of their performance on the parameters of control variables (Cho, et al., 2010). Several researches clarified that the plasma actuators could also alter the flow field for the cylinder wake (Thomas, et al., 2009); However the flow mechanism of the separation control which include the unsteady interaction between induced flow from pulsed, or unsteadily-driven plasma actuator and the separated boundary layer have not been well understood yet.

The objective of the current study is to clarify the flow mechanism of the separation control on a circular cylinder when pulsed plasma actuator is applied. At the first half of the paper, the characteristics of the induced jet from the pulsed plasma actuator are examined from the results of jet thrust measurement and high-speed schlieren flow 
visualization. Then at the second half of the paper, the effect of the frequency of pulsed plasma actuator on the flow separation on a circular cylinder model is discussed in the viewpoint of aerodynamic force and the flow field measured with time-resolved PIV at the low speed wind tunnel test.

\section{Plasma actuator}

The basic SDBD plasma actuator would consist of a pair of electrodes isolated by a dielectric material. One of the electrodes is exposed to the air and the other is buried in a dielectric material so never contacts to the air. The electrodes are arranged in a staggered manner that shares a single edge, as shown in Fig. 1. By applying kV-kHz order AC voltage, the single dielectric barrier discharge occur at the edge of the exposed electrode and the weakly ionized air, called plasma in this device, forms over the buried electrode. The ionized air and the electric field of the electrodes create body force that acts on the air flowing over the actuator. The body force can be tailored by the arrangement and orientation of the electrodes.

We employed the pulsed actuation to drive plasma actuator for this research. In this method, the applied voltage for plasma actuator is alternately turned on and off in a short period. The frequency of that period (modulation frequency: $f_{\text {mod }}$ ) is set from $1 \mathrm{~Hz}$ to $1000 \mathrm{~Hz}$. Duty Cycle (DC) is set as constant as 0.5 for all of the experiment shown in this paper.

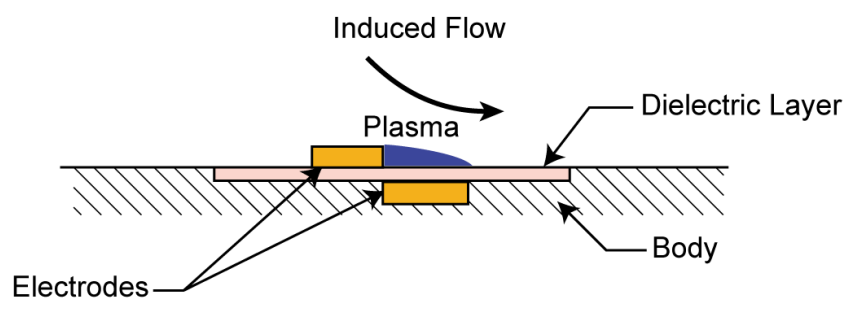

Fig. 1 Schematic of SDBD plasma actuator. The basic SDBD plasma actuator would consist of a pair of electrodes isolated by a dielectric material. By applying $\mathrm{kV}-\mathrm{kHz}$ order $\mathrm{AC}$ voltage, the single dielectric barrier discharge occurs at the edge of the exposed electrode. The ionized air and the electric field around the electrodes create body force that acts on the air flowing over the actuator.

\section{Experimental setup}

\subsection{Plasma actuator and driving apparatus}

The plasma actuator employed in this research is standard SDBD-type plasma actuator shown in Fig. 1. The configuration and the conditions of the experiment will be described in subsequent sections.

Figure 2 shows a schematic of the power supply used to generate plasma (the schematic of the setup for the wind tunnel experiment is also provided). A reference waveform of a high-voltage AC input is generated by a function generator and amplified by a solid state high power amplifier and a high voltage transformer. The sinusoidal AC input attains the amplitude up to $30 \mathrm{kV}_{\mathrm{pp}}$ at a frequency of $5-20 \mathrm{kHz}$. The voltage and current of the AC input was monitored by an oscilloscope and controlled by LabVIEW installed in a workstation.

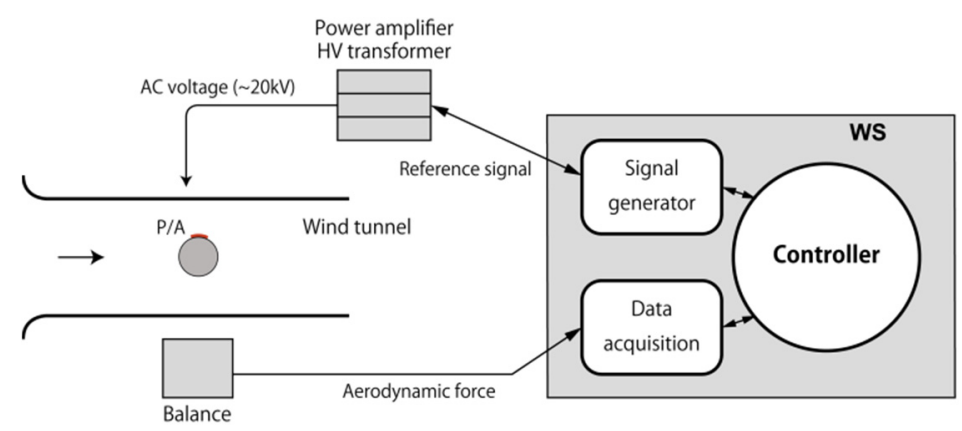

Fig. 2 Schematic diagram of the apparatus for wind tunnel experiment. A reference waveform of a high-voltage AC input is generated by a function generator and amplified by a solid state high power amplifier and a high voltage transformer. A six component external balance was employed for aerodynamic force and moment measurement. 


\subsection{Thrust and flow visualization test at the quiescent air}

In this research, the thrust of the induced jet by the plasma actuator was measured, as a reaction of the aerodynamic force from the actuator, and was evaluated as an index of the flow control performance. The thrust from plasma actuator was sensed by an analytical balance (Shimadzu AUW320), that has $<0.15 \mathrm{mg}$ in standard deviation of repeatability, with $\pm 0.3 \mathrm{mg}$ of linearity, with a lever. The uncertainty of the balance for the current experiment becomes $0.012 \mathrm{mN} / \mathrm{m}$. The schematic figure of the measurement apparatus is shown in Fig. 3.

For the current research, flow visualization has been performed using high-speed Schrielen visualization technique at the Wind Tunnel Facility of ISAS/JAXA. The Schrielen visualization has an advantage that does not require any seeding in the flow field. Although the air speed is low $(\sim 10 \mathrm{~m} / \mathrm{s})$ for the current research, Schrielen visualization is applicable. It is because that discharge generates heat and introduces heated fluid inside the induced jet, causing density gradient in flow field. Small change in density from the existence of plasma, which is generated by the discharge, may also contribute to make density gradient to some extent. With these "virtual seeding," the induced jet was able to be visualized without any external seeding.

For the high-speed Schrielen visualization, we used a high-speed camera (Phantom V7.1). Frame rate of the high-speed camera was at the rate of 800 or 3000 frame per second depending on the case.

In these experiments, the plasma actuator utilized $2 \mathrm{~mm}$ PTFE sheet as dielectric layer. Copper tape was used for the electrodes that designed $250 \mathrm{~mm}$ long and $40 \mathrm{~mm}$ wide for the buried electrode, and $5 \mathrm{~mm}$ wide for the exposed electrode. These electrodes were installed as shown in Fig.1, without any overlap between their edges.

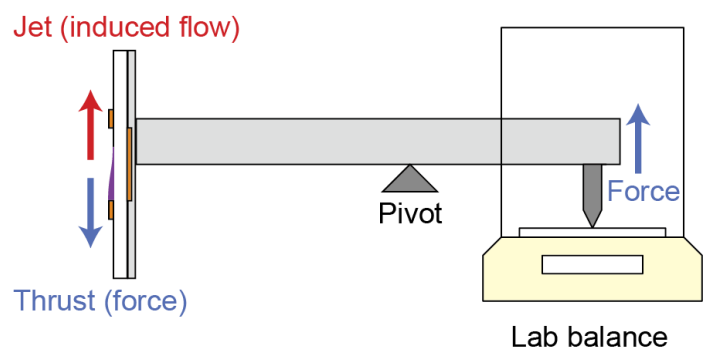

Fig. 3 Schematics of thrust measurement system. The thrust from plasma actuator was sensed by an analytical balance (Shimadzu AUW320) with a lever.

\subsection{Wind tunnel test for bluff body flow control}

The wind tunnel experiments have been made in the subsonic open-return wind tunnel in the Aerodynamics Laboratory at Tottori University. The wind tunnel has a closed test section of $0.60 \times 0.60 \mathrm{~m}$ in cross section and $1.8 \mathrm{~m}$ in length. A circular cylinder model shown in Fig. 4 was installed vertically in the test section and mounted to a six component external balance (Nissho Electric Works LMC-61187) for aerodynamic force and moment measurement. The upper end of the model is covered, and located on the tunnel wall with $5 \mathrm{~mm}$ of gap. Time-resolved PIV tests were conducted using a 1024 x 1024 pixel high speed camera (Photron FASTCAM-MAX) with a theatre fog machine for seeding and DPSS laser (Japan Laser DPGL-OL 5W) for illumination. The light sheet from the DPSS laser was aligned to illuminate the cross-section of the separation area of the cylinder model to visualize instantaneous separated flow field when the pulsed plasma actuator was driven. Acquired images were processed by the open-source particle image velocimetry software PIVLab, which developed by Thielicke et al. (Thielicke and Stamhuis, 2012 and Ryerson and Schwenk, 2011) on MATLAB. The raw image sequences were preprocessed with ImageJ, a public domain, Java-based image processing program developed at the National Institutes of Health (Rasband, 1997 and Abramoff, et al., 2004). The analysis was carried out using the image sequences taken at 2000 frames per second by the high-speed camera. The mean flow field images were calculated from 2048 pairs (one second) of their image per acquisition. To obtain the phase-averaged flow field, the acquisition was repeated at least 10 times per each condition, depending on their modulation frequency. The field-of-view is $150.2 \times 150.2 \mathrm{~mm}$ for $532 \times 532$ pixels, which leads to a resolution of 0.28 $\mathrm{mm} / \mathrm{px}$.

A plasma actuator was mounted on the surface of the model to cover the entire length of the model, with $85 \mathrm{deg}$ of 
the mount angle from the stagnation line. In this experiment, the thickness of the dielectric layer was configured as $1 \mathrm{~mm}$ thick PMMA. Other dimension of the actuator was identical to the one that was used in the thrust measurement. The uniform flow velocity for the parametric study for predominant variables was set at $U=5.0 \mathrm{~m} / \mathrm{s}$ (the Reynolds number based on the diameter of the model becomes $R e=35000)$.

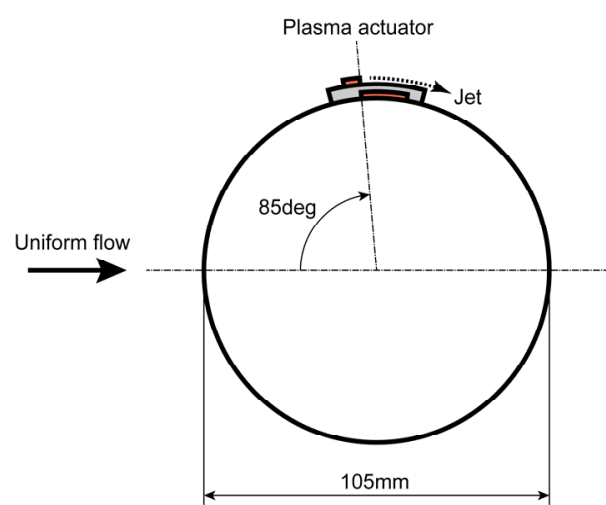

Fig. 4 Circular cylinder model and the location of plasma actuator. A plasma actuator was mounted on the surface of the model to cover the entire length of the model, with $85 \mathrm{deg}$ of the mount angle from the stagnation line.

\section{Results and Discussion}

\subsection{Flow and thrust characteristics of the pulsed plasma actuator in the quiescent air}

Figure 5 shows the thrust measured by the balance for various modulation frequencies at $V_{\mathrm{ac}}=20.6 \mathrm{kV}$ pp $(\mathrm{DC}=$ 0.5). The thrust measured is a component of the body force along with the surface of the actuator element. The data represent that the thrust is widely varied with the modulation frequency. At low frequency regime $\left(f_{\text {mod }}<250 \mathrm{~Hz}\right)$, the thrust is relatively small, compared with the high frequency cases, and shows complex characteristics. At the higher frequency regime $\left(f_{\text {mod }}>250 \mathrm{~Hz}\right)$, the thrust increases with the modulation frequency increased. We should note that the modulation frequency that produces maximum thrust, and the amount of the thrust could vary if the different driving conditions were applied, such as the high voltage AC voltage or its frequency.

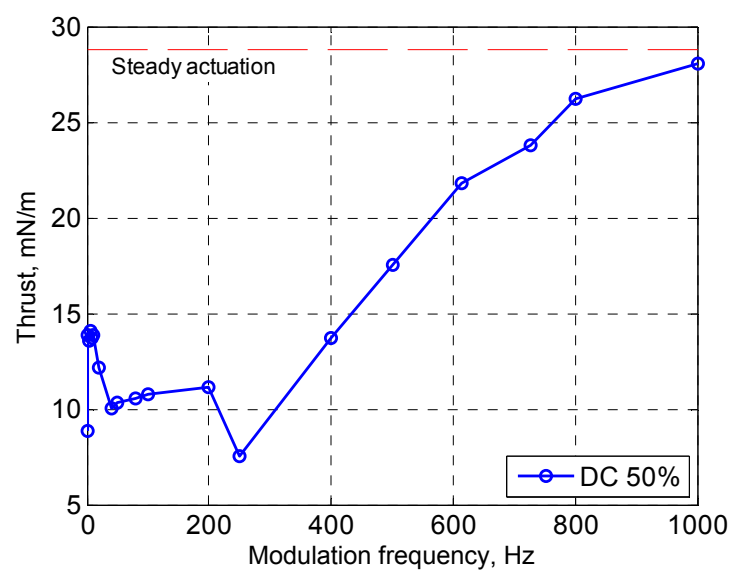

Fig. 5 Thrust vs modulation frequency at $V_{\mathrm{ac}}=20.6 \mathrm{kV}_{\mathrm{pp}}$ when the duty cycle of the pulsed actuation is $0.5(50 \%)$.

Figure 6(a)-(g) show the instantaneous images of the induced flow from the plasma actuator, taken by the high-speed schlieren visualization at $V_{\mathrm{ac}}=20.6 \mathrm{kV}_{\mathrm{pp}}$. Since the induced flow of the pulsed drive is unsteady, the images shown here only represent the flow at the instance when the plasma actuator is starting to drive (the phase in the modulation period is $0 \mathrm{deg}$ ) during their pulsed drive, except the steady case. The location of the exposed electrode is 


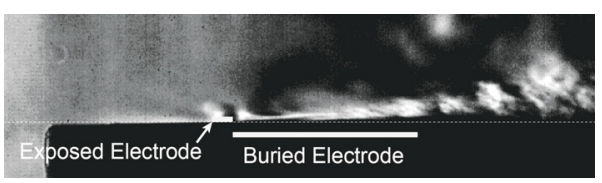

(a) Steady

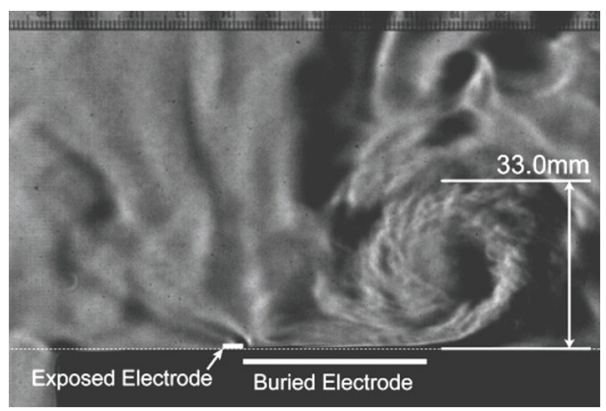

(b) $f_{\text {mod }}=1 \mathrm{~Hz}$

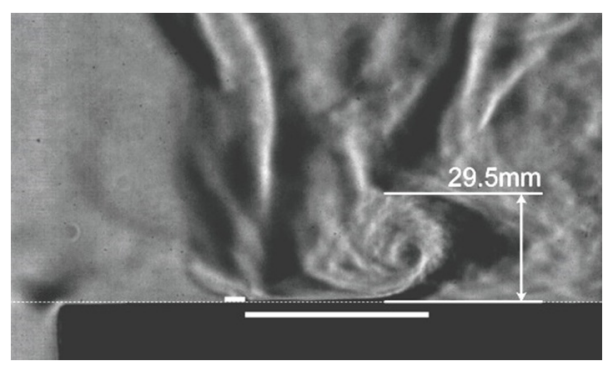

(c) $f_{\text {mod }}=10 \mathrm{~Hz}$

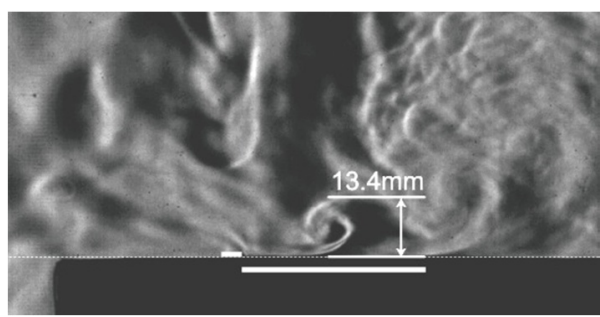

(d) $f_{\text {mod }}=40 \mathrm{~Hz}$

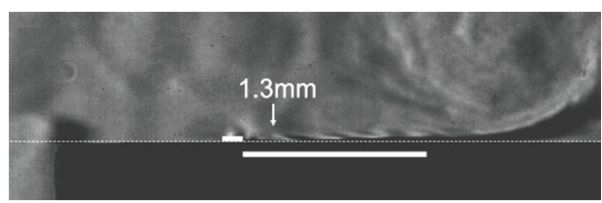

(e) $f_{\text {mod }}=400 \mathrm{~Hz}$

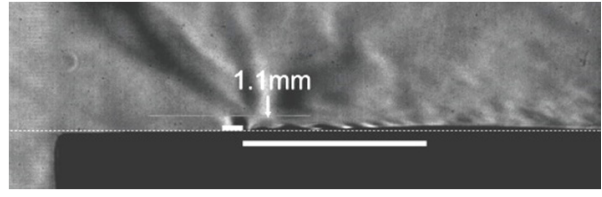

(f) $f_{\text {mod }}=615 \mathrm{~Hz}$

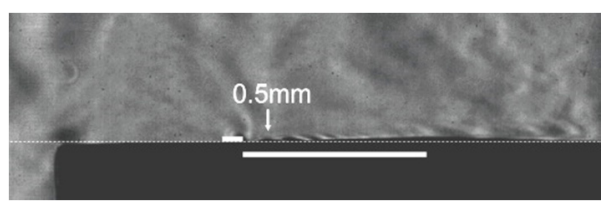

(g) $f_{\text {mod }}=1000 \mathrm{~Hz}$

Fig. 6 Instantaneous image of induced jet for unsteady actuation at $V_{\mathrm{ac}}=20.6 \mathrm{kV}_{\mathrm{pp}}$. Diameter of the vortices is superimposed on the images. The location of the exposed electrode is marked in each image.

indicated in each image. The visualized flow shows that spanwise vortices are formed by the pulsed drive. The vortices are induced by the impulsive start of the jet from the exposed electrode to the quiescent air.

The flow topology and diameter of the vortices, which was measured when the each actuation period was closed, are strongly dependent to the modulation frequency. At low modulation frequency $\left(f_{\bmod } \leq 10 \mathrm{~Hz}\right)$ the vortex is generated as isolated from the previous one. When $f_{\text {mod }}$ is beyond $10 \mathrm{~Hz}$, the induced vortex interact to the precedent one and resulted regularly aligned vortex sheet appears over the large area of the downstream. At these regimes, the area that covers the downstream of the actuator becomes small, so that thin induced jet that has spanwise vortex is formed. Beyond $f_{\text {mod }}=400 \mathrm{~Hz}$, thin and wall-bounded jet is generated. The jet at this condition is thinner than the steadily-actuated case (at least for the fluid element that is influenced by the plasma around the exposed electrode). For these cases the diameter of the vortices is remarkably small, and finally almost invisible from the images shown here.

Figure 7 show the time history of convective velocity of the induced vortices for each modulation frequency. The convective velocity of the induced vortex was measured from the time-resolved Schlieren images, by identifying the locations of center of the induced vortex. The images were processed from the time when the plasma actuator is start to operate, and trace them until the center of the vortex reaches on the aft edge of the buried electrode (40mm from the exposed electrode).

The vortex convective velocity was supposed to represent the magnitude of their jet velocity, which is difficult to determine for unsteady actuation discussed in this paper. Commonly for these cases, the vortex convective velocity is high, around $5 \mathrm{~m} / \mathrm{s}$, at $\mathrm{t} \sim 0$ when the actuator start to drive. They are immediately decelerated in several milliseconds to around $1 \mathrm{~m} / \mathrm{s}$, and continue to move with this velocity. At the higher modulation frequency $\left(f_{\text {mod }}>400 \mathrm{~Hz}\right)$, the convective velocity maintains its initial value, around $5 \mathrm{~m} / \mathrm{s}$, until the vortex arrives the aft end of the buried electrode. This suggests that the induced flow at high modulation frequency has strong directivity and could keep their jet strong. These observations are consistent with the thrust characteristics shown in Fig. 5. 


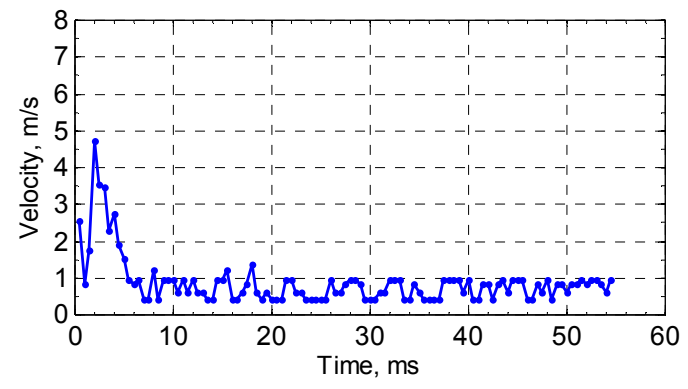

(a) $f_{\text {mod }}=1 \mathrm{~Hz}$

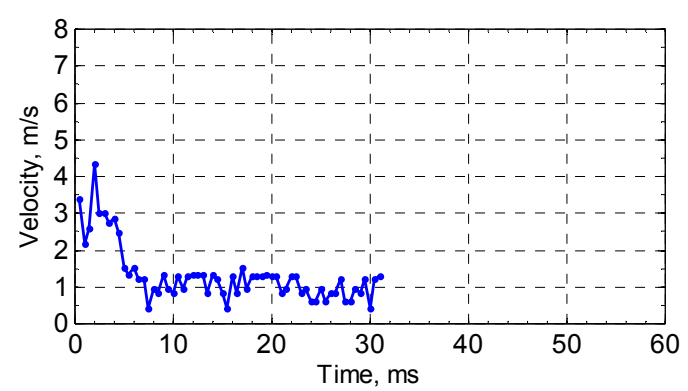

(b) $f_{\text {mod }}=40 \mathrm{~Hz}$

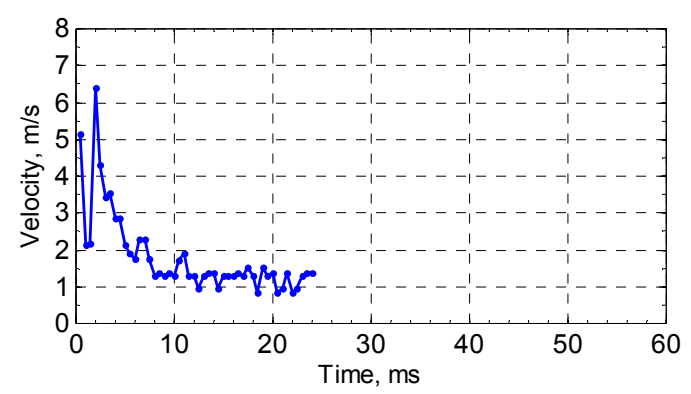

(c) $f_{\bmod }=100 \mathrm{~Hz}$

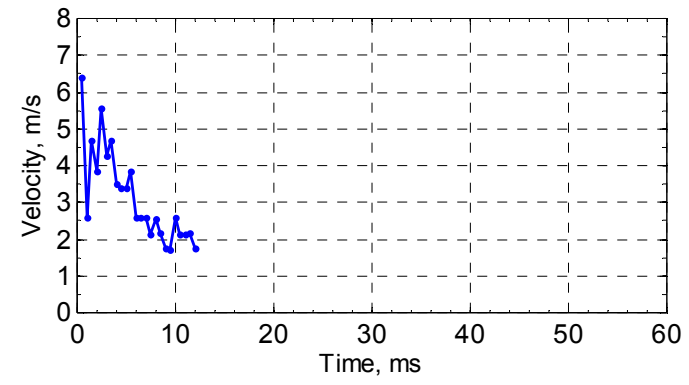

(d) $f_{\text {mod }}=400 \mathrm{~Hz}$

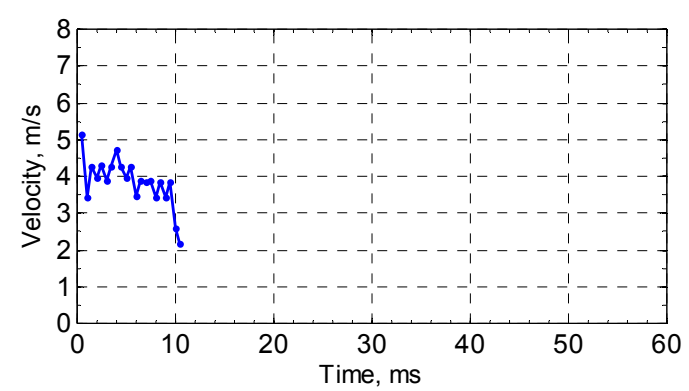

(e) $f_{\bmod }=615 \mathrm{~Hz}$

Fig. 7 Time history of the vortex convective velocity for several modulation frequency at $V_{\mathrm{ac}}=20.6 \mathrm{kV}$. The vortex convective velocity was measured from the image sequences of the high-speed schlieren visualization.

\subsection{Bluff body flow control}

In this section, the effect of pulsed plasma actuator to the separation from the circular cylinder model is discussed. Figure 8 shows the drag coefficient of the circular cylinder model when this pulsed actuation is applied to the actuator with several modulation frequencies. For these cases, the applied voltage was fixed at $V_{\mathrm{ac}}=21 \mathrm{kV}$ pp with the $14 \mathrm{kHz}$ of their frequency. The drag coefficient when the plasma actuator was turned off is plotted at $f_{\bmod }=0 \mathrm{~Hz}$ for comparison. The drag is significantly dropped, and slightly decreased with increasing the modulation frequency, except when $f_{\text {mod }}=$ $10 \mathrm{~Hz}$. At low frequency regime, the characteristics might be more complex with a strong dependency to the modulation frequency. On the other hand, at higher frequency the general trend should be same beyond $f_{\text {mod }}=250 \mathrm{~Hz}$, up to $500 \sim 800 \mathrm{~Hz}$. 


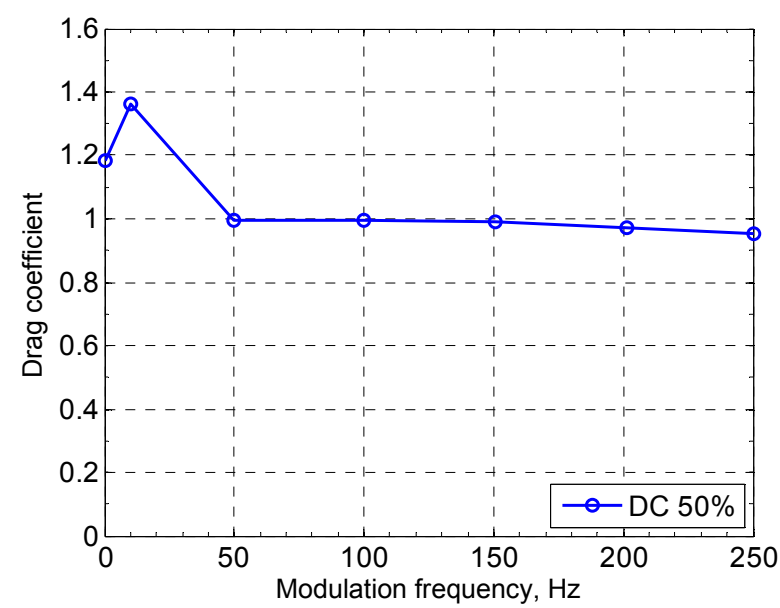

Fig. 8 Drag coefficient vs modulation frequency for circular cylinder model at $V_{\mathrm{ac}}=21 \mathrm{kV}$ pp. The applied voltage was set at $V_{\mathrm{ac}}=21 \mathrm{kV}_{\mathrm{pp}}$ with the $14 \mathrm{kHz}$ of their frequency. The drag coefficient when the plasma actuator was turned off is plotted at $f_{\text {mod }}=0 \mathrm{~Hz}$ for comparison.

The mean velocity field was obtained by time-averaging the time-resolved PIV data. Figure 9(a)-(d) show the mean velocity vector field of the separated area behind the circular cylinder model. The corresponding time-averaged absolute velocity contours are also plotted in this figure. As expected, the separation was suppressed and resulting wake area was reduced by applying plasma actuator. From the time-averaged results, the location of the separation point (note that it was defined by the direction of the velocity vector which is the closest to the model surface in this paper) moves downstream as $f_{\text {mod }}$ increase. This should directly correspond to the dependency of drag coefficient on the modulation frequency shown in Fig. 8. However, even at $f_{\text {mod }}=10 \mathrm{~Hz}$, the time-averaged flow field shows significant reduction of the wake, that is inconsistent with aerodynamic data.

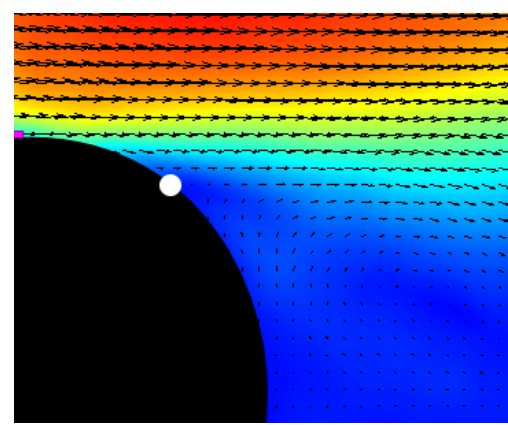

(a) Off

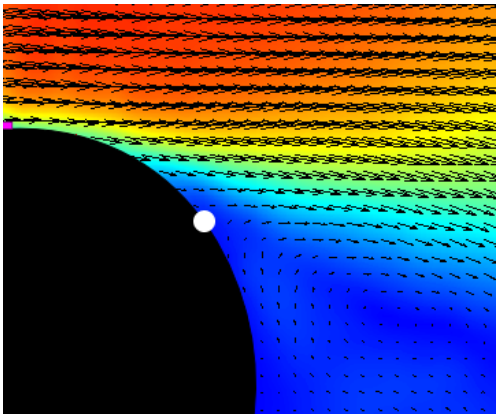

(b) $f_{\text {mod }}=10 \mathrm{~Hz}$

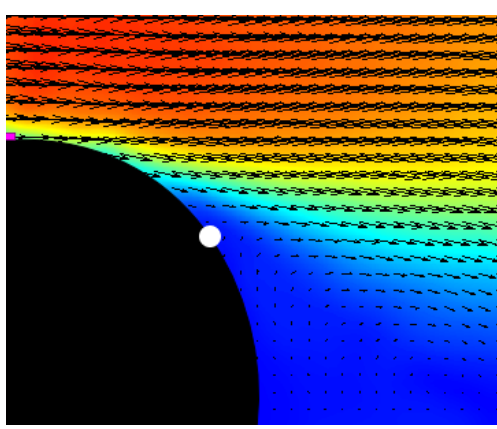

(c) $f_{\text {mod }}=100 \mathrm{~Hz}$

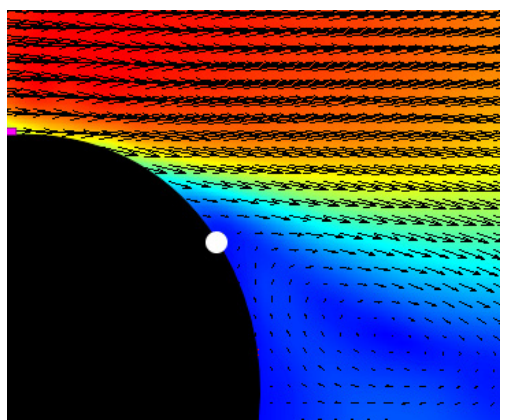

(d) $f_{\bmod }=200 \mathrm{~Hz}$

\begin{tabular}{|l|}
\hline Vabs \\
5.5 \\
5 \\
4.5 \\
4 \\
3.5 \\
3 \\
2.5 \\
2 \\
1.5 \\
1 \\
0.5 \\
0 \\
\hline
\end{tabular}

Fig. 9 Time-averaged velocity field behind the circular cylinder model at $V_{\mathrm{ac}}=21 \mathrm{kV}$ pp. The corresponding time-averaged absolute velocity contours are also plotted. The location of the separation point is plotted as white dot. 
Figures 10 (a) (c) shows the phase averaged velocity vector fields of the separated area behind the circular cylinder model for each modulation frequency $\left(f_{\bmod }=10,100\right.$ and $200 \mathrm{~Hz}$ respectively). The phase-averaged fields were processed from the time-resolved PIV data which are same as Fig. 9. Phase averaging has been carried out using $f_{\text {mod }}$ for the base frequency. In these plots, the plasma actuator was turned on at the phase $\phi=0 \%$, and turned off at $\phi=50 \%$ since the duty cycle of the pulsed actuation was set at 0.5 for these tests.

At $f_{\text {mod }}=10 \mathrm{~Hz}$, the location of the separation point varies widely with the plasma actuation in each cycle. Their motion has a significant time lag in this case, such that the separation point reaches the aft end when $\phi=40 \%$. In the current experimental conditions, the Strouhal number of the wake vortex fluctuation is known as 0.2 , which corresponds $9.52 \mathrm{~Hz}$ in frequency. Therefore in this case the plasma actuation accelerates the fluctuation of the wake vortex, and resulting aerodynamic drag becomes large as shown in Fig. 8. On the contrary, with $f_{\text {mod }}=100$ and $200 \mathrm{~Hz}$, the flow around the cylinder is stabilized. In these cases, we did not find large fluctuation which related with the modulation frequency in the flow around the cylinder, as well as the separation point. This is also represented from the result at $f_{\text {mod }}=10 \mathrm{~Hz}$ that the time lag in the movement of the separation point has relatively large time scale compared to these $(100 \sim 200 \mathrm{~Hz})$ frequency range. In addition to that, the flow field on the lower side (the side that the plasma actuator is not installed) should have an effect on the aerodynamic control performance in these conditions. Especially at $f_{\text {mod }}=10 \mathrm{~Hz}$, the separated flow on the lower side will be similar characteristics to the flow on the upper side as shown in Fig. 10 (a), because the drive of the plasma actuator accelerates the fluctuation of the separated wake vortices.

From these results, the main cause of the enhancement of drag reduction performance in these cases would be the enhancement of the induced jet generated from plasma actuator as shown in Fig. 5. This is confirmed by Fig. 10 (b) and Fig. 10 (c), showing the absolute velocity over the cylinder improved as the modulation frequency is increased.

\section{Summary}

To clarify the flow mechanism of the separation control on a circular cylinder when pulsed plasma actuator is applied, the characteristics of the induced jet from the pulsed plasma actuator were examined and low speed wind tunnel testing was carried out. For the induced jet analysis, the strength of the induced jet is found to be widely varied with the modulation frequency. When the modulation frequency is high $\left(f_{\bmod }>250 \mathrm{~Hz}\right)$, the thrust of the induced jet increases with the modulation frequency increased. In this condition, thin and wall-bounded jet is generated. For the separation control test, the drag is significantly dropped, and gradually decreased with increasing the modulation frequency. The drag increased when $f_{\text {mod }}=10 \mathrm{~Hz}$. From the results of time-resolved PIV, we found that this is because the plasma actuation accelerates the natural fluctuation of the wake vortex. The main cause of the enhancement of drag reduction performance in high $f_{\text {mod }}$ regime would be the enhancement of the induced jet generated from plasma actuator. 


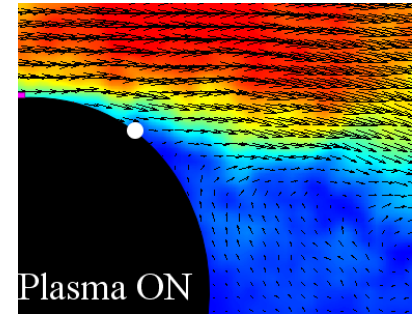

$\phi=0 \%$

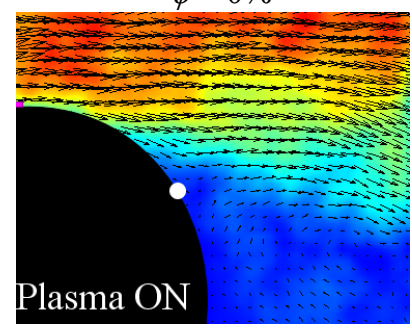

$\phi=20 \%$

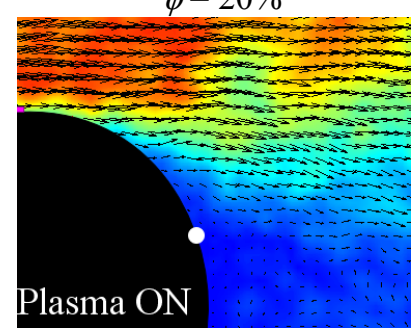

$\phi=40 \%$

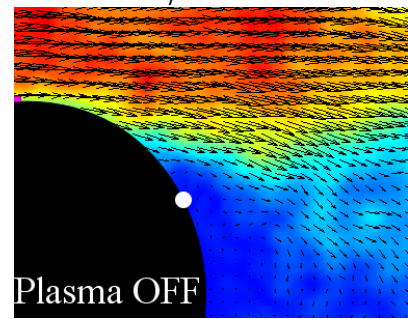

$\phi=60 \%$

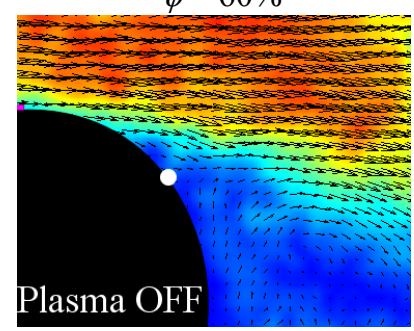

$\phi=80 \%$

(a) $f_{\bmod }=10 \mathrm{~Hz}$

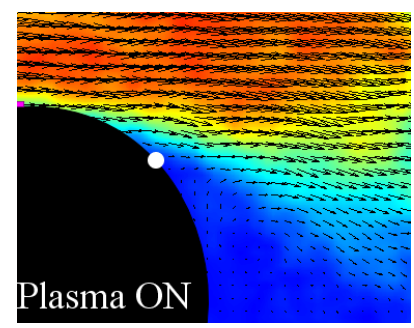

$\phi=0 \%$

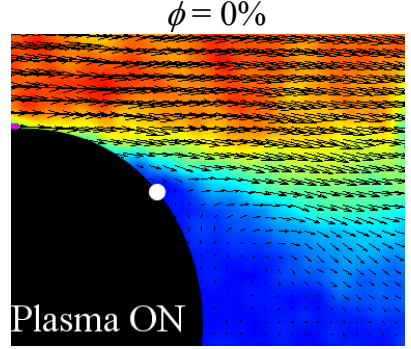

$\phi=20 \%$

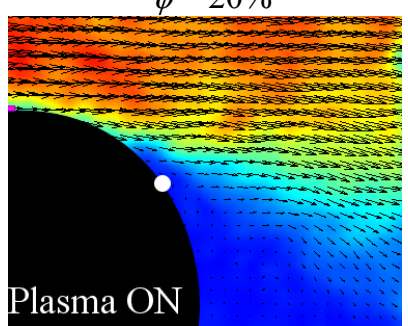

$\phi=40 \%$

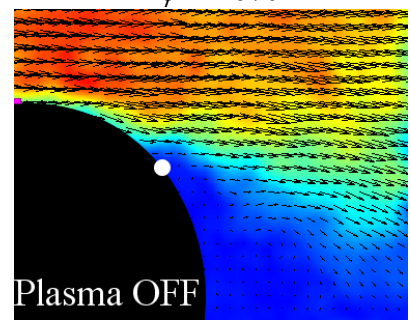

$\phi=60 \%$

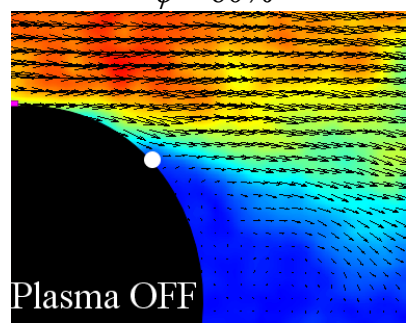

$\phi=80 \%$

(b) $f_{\text {mod }}=100 \mathrm{~Hz}$

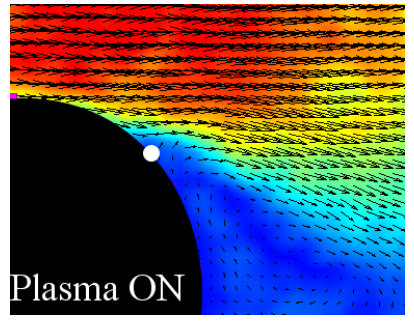

$\phi=0 \%$

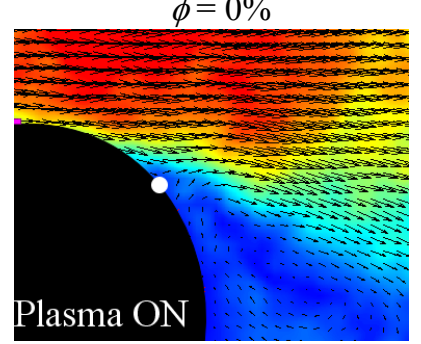

$\phi=20 \%$

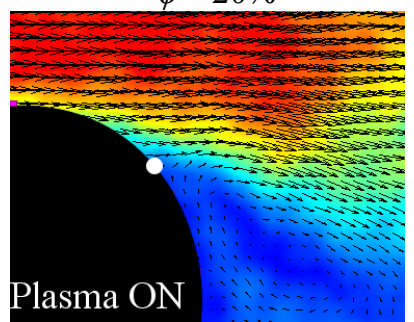

$\phi=40 \%$

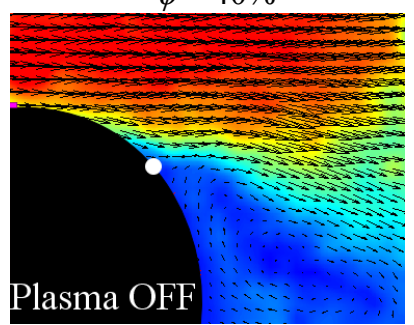

$\phi=60 \%$

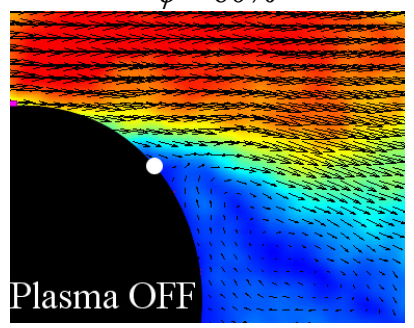

$\phi=80 \%$

(c) $f_{\bmod }=200 \mathrm{~Hz}$

Fig. 10 Phase-averaged velocity field behind the circular cylinder model for various modulation frequencies.

\section{Acknowledgment}

This research was partially supported by KAKENHI (19686051, 23760771) from JSPS, JAPAN. We wish to acknowledge the cooperation of Prof. Kozo Fujii, Prof. Akira Oyama, Dr. Taku Nonomura and their colleagues for conducting high-speed Schrielen visualization at the ISAS/JAXA. We also thank Mr. Teruaki Baba for his help during the course of this study. 


\section{References}

Abramoff, M. D., Magalhaes, P. J. and Ram, S. J., Image processing with ImageJ, Biophotonics International, Vol. 11, Issue 7 (2004), pp. 36-42.

Cho, Y. C., Jayaraman, B., Viana, F., Haftka, R. and Shyy, W., Surrogate modelling for characterising the performance of a dielectric barrier discharge plasma actuator, International Journal of Computational Fluid Dynamics, Vol. 24, Issue 7 (2010), pp. 281-301.

Corke, T. C., Post, M. L. and Orlov, D. M., SDBD plasma enhanced aerodynamics: concepts, optimization and applications, Progress in Aerospace Sciences, Vol.43 (2007), pp. 193-217.

Matsuno, T., Maeda, K., Yamada, G., Kawazoe, H. and Kanazaki, M., Improvement of flow control performance of plasma actuator using wind-tunnel test based Efficient Global Optimization, AIAA paper, No. 2013-2512 (2013).

Matsuno, T., Separation control using plasma actuators, Journal of the Japan Society of Mechanical Engineers, Vol. 115, No. 1127 (2012), pp. 692-696 (in Japanese).

Matsuno, T., Kawaguchi, M., Yamada, G. and Kawazoe, H., Development of trielectrode plasma actuator and its application to delta wing vortex control. AIAA paper, No. 2011-3514 (2011).

Matsuno, T., Ota, K., Kanatani, T. and Kawazoe, H., Parameter design optimization of plasma actuator configuration for separation control. AIAA Paper, No. 2010-4983 (2010).

Ryerson, W. G. and Schwenk, K., A simple, inexpensive system for digital particle image velocimetry (dpiv) in biomechanics, Journal of Experimental Zoology Part A: Ecological Genetics and Physiology, Vol. 317. Issue 2 (2011), pp. 127-140.

Rasband, W. S., ImageJ, Software Package, Version 1.46p, U. S. National Institutes of Health, Bethesda, Maryland, USA, available from $<$ http://imagej.nih.gov/ij/>, (accessed on 30 January, 2014)

Segawa, T., Furutani, H, Abe, H., Yoshida, H., Takekawa, S. and Choi, K.-S., Active control of separation flow around airfoil using DBD plasma actuator, Theoretical and Applied Mechanics Japan, Vol. 59 (2011), pp. 265-274.

Thielicke, W. and Stamhuis, E. J., PIVlab - time-resolved particle image velocimetry (PIV) tool, Software Package, Version 1.31, available from <http://pivlab.blogspot.jp/> , (accessed on 30 January, 2014).

Thomas, F. O., Corke, T., C., Iqbal, M., Kozlov, A. and Schatzman, D., Optimization of dielectric barrier discharge plasma actuators for active aerodynamic flow control, AIAA Journal, Vol. 47 (2009), pp. 2169-2178. 